\title{
DISCOVERY, PROGENITOR AND EARLY EVOLUTION OF A STRIPPED ENVELOPE SUPERNOVA iPTF13bvn
}

\author{
Yi CaO ${ }^{1}$, Mansi M. Kasliwal ${ }^{2}$, Iair Arcavi ${ }^{3}$, Assaf Horesh ${ }^{1}$, Paul Hancock Pa $^{4,5}$, Stefano Valenti $^{6,7}$, S. Bradley Cenko $^{8}$,
} S. R. Kulkarni ${ }^{1}$, Avishay Gal-Yam ${ }^{3}$, Evgeny Gorbikov ${ }^{3}$, Eran O. Ofek ${ }^{3}$, David Sand ${ }^{9}$, Ofer Yaron $^{3}$, Melissa Graham ${ }^{6,7}$, Jeffrey M. Silverman ${ }^{10}$, J. Craig Wheeler ${ }^{10}$, G. H. Marion ${ }^{10}$, Emma S. Walker ${ }^{11}$, Paolo Mazzali ${ }^{12,13,14}$, D. Andrew Howell ${ }^{6,7}$, K. L. Li ${ }^{15}$, A. K. H. Kong ${ }^{15}$, Joshua S. Bloom ${ }^{16}$, Peter E. Nugent ${ }^{16,17}$, Jason Surace ${ }^{18}$,

Frank Masci $^{19}{ }^{\text {, John Carpenter }}{ }^{1}$, Nathalie DegenaAR ${ }^{20}$, and Christopher R. Gelino ${ }^{19}$

${ }^{1}$ Astronomy Department, California Institute of Technology, 1200 E. California Boulevard, Pasadena, CA 91125, USA; ycao@ astro.caltech.edu

${ }^{2}$ The Observatories, Carnegie Institution for Science, 813 Santa Barbara Street, Pasadena, CA 91101, USA

${ }^{3}$ Department of Particle Physics and Astrophysics, Weizmann Institute of Science, Rehovot 76100, Israel

${ }^{4}$ Sydney Institute for Astronomy (SIfA), School of Physics, The University of Sydney, NSW 2006, Australia

${ }^{5}$ ARC Centre of Excellence for All-sky Astrophysics (CAASTRO), The University of Sydney, NSW 2006, Australia

${ }^{6}$ Las Cumbres Observatory Global Telescope Network, Goleta, CA 93117, USA

${ }^{7}$ Department of Physics, University of California, Santa Barbara, CA 93106, USA

${ }^{8}$ Astrophysics Science Division, NASA Goddard Space Flight Center, Mail Code 661, Greenbelt, MD 20771, USA

${ }^{9}$ Department of Physics, Texas Tech University, Lubbock, TX 79409, USA

${ }^{10}$ Department of Astronomy, University of Texas at Austin, Austin, TX 78712, USA

${ }_{11}$ Department of Physics, Yale University, New Haven, CT 06511-8499, USA

12 INAF-Padova Astronomical Observatory, Vicolo dell'Osservatorio 5, I-35122 Padova, Italy

${ }^{13}$ Astrophysics Research Institute, Liverpool John Moores University, Liverpool, UK

${ }^{14}$ Max-Planck Institute for Astrophysics, D-85748 Garching, Germany

${ }^{15}$ Institute of Astronomy and Department of Physics, National Tsing Hua University, Hsinchu 30013, Taiwan

${ }^{16}$ Department of Astronomy, University of California Berkeley, B-20 Hearst Field Annex \# 3411, Berkeley, CA 94720-3411, USA

${ }^{17}$ Computational Cosmology Center, Computational Research Division, Lawrence Berkeley National Laboratory, 1 Cyclotron Road MS 50B-4206, Berkeley, CA 94720, USA

${ }^{18}$ Spitzer Science Center, MS 220-6, California Institute of Technology, Jet Propulsion Laboratory, Pasadena, CA 91125, USA

${ }^{19}$ Infrared Processing and Analysis Center, California Institute of Technology, Pasadena, CA 91125, USA

${ }^{20}$ Department of Astronomy, University of Michigan, 500 Church Street, Ann Arbor, MI 48109, USA Received 2013 July 4; accepted 2013 August 15; published 2013 August 30

\begin{abstract}
The intermediate Palomar Transient Factory reports our discovery of a young supernova, iPTF13bvn, in the nearby galaxy, NGC 5806 (22.5 Mpc). Our spectral sequence in the optical and infrared suggests a Type Ib classification. We identify a blue progenitor candidate in deep pre-explosion imaging within a $2 \sigma$ error circle of $80 \mathrm{mas}(8.7 \mathrm{pc})$. The candidate has an $M_{B}$ luminosity of $-5.52 \pm 0.39$ mag and a $B-I$ color of $0.25 \pm 0.25$ mag. If confirmed by future observations, this would be the first direct detection for a progenitor of a Type Ib. Fitting a power law to the early light curve, we find an extrapolated explosion date around 0.6 days before our first detection. We see no evidence of shock cooling. The pre-explosion detection limits constrain the radius of the progenitor to be smaller than a few solar radii. iPTF13bvn is also detected in centimeter and millimeter wavelengths. Fitting a synchrotron self-absorption model to our radio data, we find a mass-loading parameter of $1.3 \times 10^{12} \mathrm{~g} \mathrm{~cm}^{-1}$. Assuming a wind velocity of $10^{3} \mathrm{~km} \mathrm{~s}^{-1}$, we derive a progenitor mass-loss rate of $3 \times 10^{-5} M_{\odot} \mathrm{yr}^{-1}$. Our observations, taken as a whole, are consistent with a Wolf-Rayet progenitor of the supernova iPTF13bvn.
\end{abstract}

Key words: instrumentation: adaptive optics - shock waves - stars: Wolf-Rayet - supernovae: individual (iPTF13bvn) - surveys

Online-only material: color figures

\section{INTRODUCTION}

Supernovae of Type $\mathrm{Ib} / \mathrm{c}$ constitute about one-third of the death rate of massive stars ( $\mathrm{Li}$ et al. 2011). Their spectra lack hydrogen (Filippenko 1997), suggesting progenitors stripped of their hydrogen envelopes either due to mass transfer in a binary system or via copious stellar winds. On theoretical grounds, the anticipated progenitors are Wolf-Rayet (WR) stars or massive helium stars (e.g., Dessart et al. 2012; Yoon et al. 2012).

The most direct way to test the above theoretical picture is direct detection of the progenitor. This is possible for supernovae occurring in nearby galaxies which have deep pre-explosion images (which means, in practice, Hubble Space Telescope, HST). Eldridge et al. (2013) comprehensively summarize searches for $12 \mathrm{Ib} / \mathrm{c}$ progenitors, which are all non-detections. The deepest upper limit to date is $M_{B}=-4.4 \mathrm{mag}$ for SN 2002ap, a Type Ic supernova in the very nearby galaxy Messier 74 (Crockett et al. 2007).
An indirect way to infer the progenitor properties is the early light curve of a supernova, which is sensitive to the progenitor size, explosion energy, and the composition of the outer layers (e.g., Nakar \& Sari 2010; Rabinak \& Waxman 2011; Bersten et al. 2012; Piro \& Nakar 2013), and in some propitious cases can even diagnose the presence of a binary companion (Kasen 2010). No detection of shock cooling constrains the radius of PTF10vgv (Type Ic) to $<6 R_{\odot}$ (Corsi et al. 2012; Piro \& Nakar 2013). For SN 2008D (Type Ib), the shock cooling phase lasted about five days and the inferred radius is $<14 R_{\odot}$ (Soderberg et al. 2008).

Radio measurements serve as another indirect probe of the progenitor system by characterizing the mass-loss history. Radio emission is produced by relativistic electrons accelerated in the supernova shock as they gyrate in the amplified magnetic field when the shock expands freely. The radio spectral evolution implies a mass-loading parameter $A=\dot{M} /\left(4 \pi v_{w}\right)$, where $\dot{M}$ is a constant mass-loss rate and $v_{w}$ is the wind velocity from 


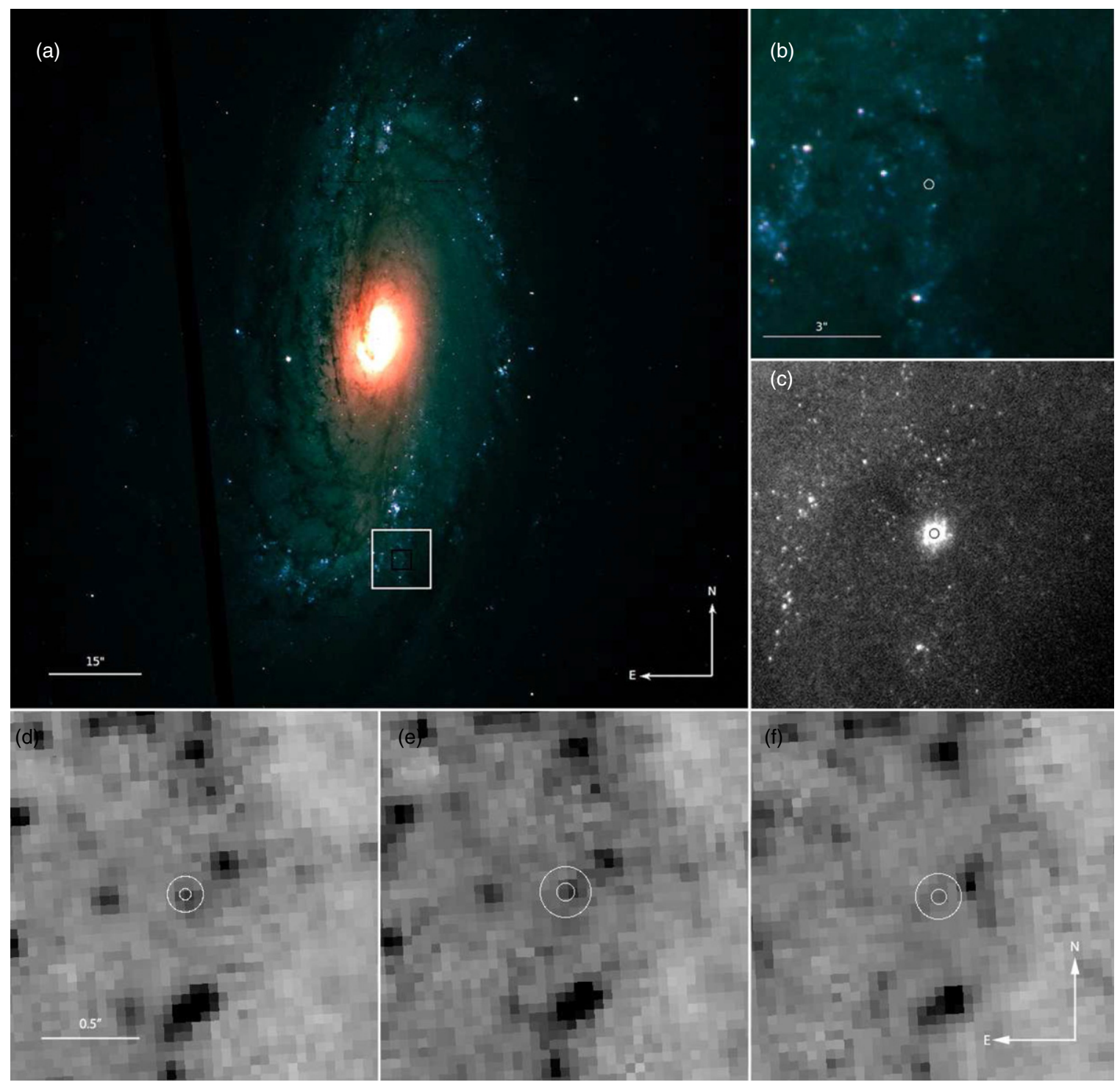

Figure 1. Supernova iPTF13bvn and its host galaxy, NGC 5806. The supernova is located at $\alpha=15^{\mathrm{h}} 00^{\mathrm{m}} 00^{\mathrm{s}} .152, \delta=+01^{\circ} 52^{\prime} 53^{\prime \prime} .17$ (J2000). Panel (a) shows the $H S T$ image of NGC 5806, taken on 2005 March 10 UT (HST proposal 10187, PI: Smartt). Panel (b) is a zoom-in of the HST image near the site of iPTF13bvn. Panel (c) is the composite image of the supernova taken with OSIRIS and LGS-AO system. This is made by stacking $6015 \mathrm{~s}$ exposures. We align this image with the HST/ACS images with 15, 23, and 25 registration stars in the F435W, F555W, and F814W filters and acquire registration uncertainties of $0.6,0.9$, and 0.8 HST pixel, respectively. Panels (d), (e), and (f) show the HST images at the position of the supernova in F435W, F555W, and F814W filters. The position of iPTF13bvn is marked with $1 \sigma$ and $3 \sigma$ error circles. The progenitor candidate is $26.50 \pm 0.15 \mathrm{mag}$ in $\mathrm{F} 435 \mathrm{~W}(\mathrm{~S} / \mathrm{N}=11.2), 26.40 \pm 0.15 \mathrm{mag}$ in F555W ( $\mathrm{S} / \mathrm{N}=9.9)$, and 26.10 $\pm 0.20 \mathrm{mag}$ in $\mathrm{F} 814 \mathrm{~W}$ $(\mathrm{S} / \mathrm{N}=8.2)$.

(A color version of this figure is available in the online journal.)

the progenitor (Chevalier 1998). Type Ib/c supernovae typically have $A$ of the order of $10^{12} \mathrm{~g} \mathrm{~cm}^{-1}$ (Chevalier \& Fransson 2006).

In this Letter, we report the discovery of iPTF13bvn, a young Type Ib supernova in the nearby galaxy NGC 5806. Archival HST images of NGC 5806 allow a direct progenitor search. We also present early photometric, spectroscopic, and wide-band radio observations and discuss implications on the progenitor.

\section{DISCOVERY}

On 2013 June 16.238 UT, ${ }^{21}$ the automated real-time discovery and classification pipeline of the intermediate Palomar Transient Factory (iPTF; Law et al. 2009; P. E. Nugent et al., in preparation) identified a new transient source with

\footnotetext{
${ }^{21}$ All times are in UT.
}

$r=18.6 \mathrm{mag}^{22}$ in the vicinity of NGC 5806 (see Figure 1; Cao et al. 2013). No source was detected at the same location to $<21.7 \mathrm{mag}(3 \sigma)$ on June 15.240 (Figure 2). Also, there was no evidence for pre-outburst activity in 492 PTF images taken since 2009 June 29 to a similar depth. Our duty astronomer saved this source as iPTF13bvn and initiated rapid, multi-wavelength follow-up.

\section{PROGENITOR IDENTIFICATION}

On June 20.276, we observed iPTF13bvn in $H$-band with OSIRIS (Larkin et al. 2006) and the Laser Guide Star Adaptive Optics (LGS-AO) system (Wizinowich et al. 2006) mounted on the $10 \mathrm{~m}$ Keck I telescope. Registering the AO image to

\footnotetext{
${ }^{22}$ BVRI mags are in Vega; all other mags are in $\mathrm{AB}$ system.
} 

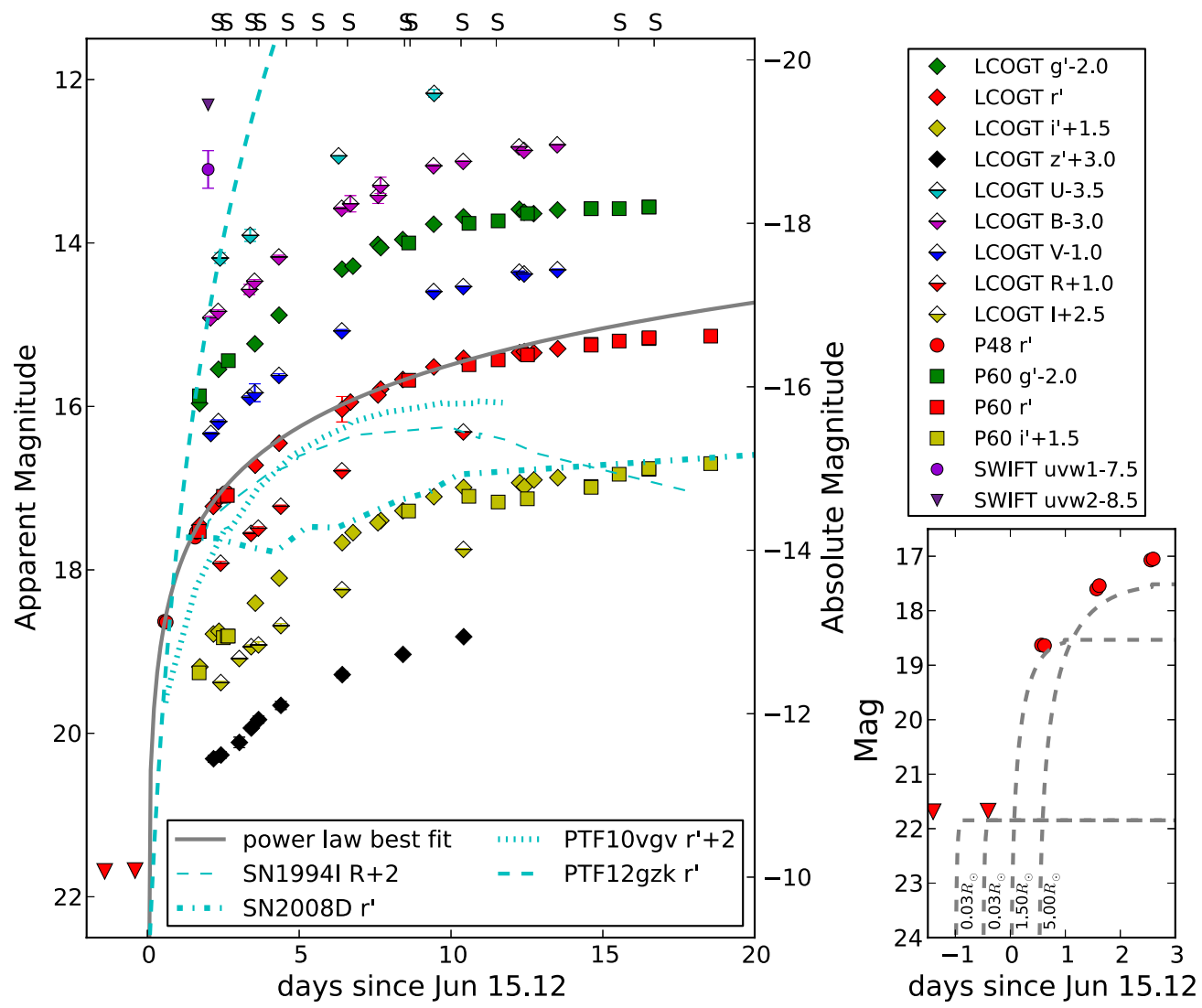

Figure 2. Left panel: multi-band light curve of iPTF13bvn (the color represents the filter, and the symbol shape represents the telescope). The upper limits are denoted in triangles. The origin $t=0$ is set to the explosion date derived from the best power-law fit (solid gray curve). For comparison, $r$-band light curves of SN 1994I, SN 2008D, PTF10vgv, and PTF12gzk are also shown in cyan with different line styles. On the top axis, the epochs of spectroscopic follow-up are indicated by "S." In the right panel, the P48 $r$-band light curves are plotted against predicted light curves of shock cooling (gray dash curves) from Piro \& Nakar (2013) for explosions starting at $t=-1,-0.5,0.0$, and 0.5 days with a "mean" velocity of $8000 \mathrm{~km} \mathrm{~s}^{-1}$. The radius of the progenitor in each of the models is besides its corresponding light curve.

(A color version of this figure is available in the online journal.)

the archival $H S T /$ ACS image, we obtain a $1 \sigma$ uncertainty of $\lesssim 40$ mas. We find one source coincident with the supernova within the $2 \sigma$ (equivalently $8.7 \mathrm{pc}$ projection distance) error circle (Figure 1; Arcavi et al. 2013b).

We perform point-spread function (PSF) photometry on the HST/ACS images with DOLPHOT (Dolphin 2000). The photometry of the progenitor candidate is $26.50 \pm 0.15 \mathrm{mag}$ in $\mathrm{F} 435 \mathrm{~W}, 26.40 \pm 0.15 \mathrm{mag}$ in F555W, and $26.10 \pm 0.20 \mathrm{mag}$ in F814W. To correct for extinction, we obtain a high-resolution spectrum (Section 4.1) and measure the equivalent widths of Na I D lines. We find local extinction of $E(B-V)=0.0437$ and foreground extinction of 0.0278 (Poznanski et al. 2012). Assuming $R_{V}=3.1$ (Schlafly \& Finkbeiner 2011) and adopting a distance modulus of $31.76 \pm 0.36$ (22.5 Mpc; Tully et al. 2009), we find $M_{B}=-5.52 \pm 0.39 \mathrm{mag}, M_{V}=-5.55 \pm 0.39 \mathrm{mag}$, and $M_{I}=-5.77 \pm 0.41 \mathrm{mag}$. Thus, the progenitor, if single, is no brighter than these values. The luminosity and colors are consistent with the compilation of WN and WC Wolf-Rayet stars in Eldridge et al. (2013).

However, WR stars are often in binaries (Tuthill et al. 1999). Thus, the progenitor is possibly in a binary system and the light is dominated by the companion. We further note that color alone cannot be used as a discriminant. The $B, V$, and $I$ filters are in the Rayleigh-Jeans tail of hot stars, including O-stars, WR stars, and blue supergiants. Moreover, given the $0{ }^{\prime} 05$ pixel size of $H S T /$ ACS, or equivalently $5.45 \mathrm{pc}$ at the distance of NGC 5806, the candidate can also be an unresolved young star cluster whose color is dominated by OB stars. Finally, we caution that the progenitor candidate may even be unrelated to the supernova.

The litmus test of whether this candidate is the progenitor or part of the progenitor system can only be undertaken by HST imaging after the supernova fades.

\section{EARLY PHOTOMETRIC AND SPECTROSCOPIC EVOLUTION}

\subsection{Observations and Reduction}

As part of regular iPTF operations, the field of iPTF13bvn was imaged twice a night every night by the Palomar 48 inch Oschin telescope (P48) with a Mould $R$-band filter (Ofek et al. 2012) during the Spring quarter. Upon discovery of iPTF13bvn, the robotic Palomar 60 inch telescope (P60; Cenko et al. 2006) was triggered for follow-up in the $g^{\prime} r^{\prime} i^{\prime} z^{\prime}$ bands. We obtained photometry with the Las Cumbres Observatory Global Telescope (LCOGT; Brown et al. 2013) network in the UBVRI $g^{\prime} r^{\prime} i^{\prime} z^{\prime}$ bands using the $1 \mathrm{~m}$ telescopes from Cerro Tololo (Chile), McDonald Observatory (USA), and Sutherland (South Africa), along with the $2 \mathrm{~m}$ Faulkes Telescope South (Siding Springs). As part of our ongoing iPTF-Swift program, ${ }^{23}$ we triggered target-of-opportunity observations beginning on 2013 June 17 (Arcavi et al. 2013a). In the P48, P60, and

\footnotetext{
${ }^{23}$ PID 9120112, PI: Kasliwal.
} 


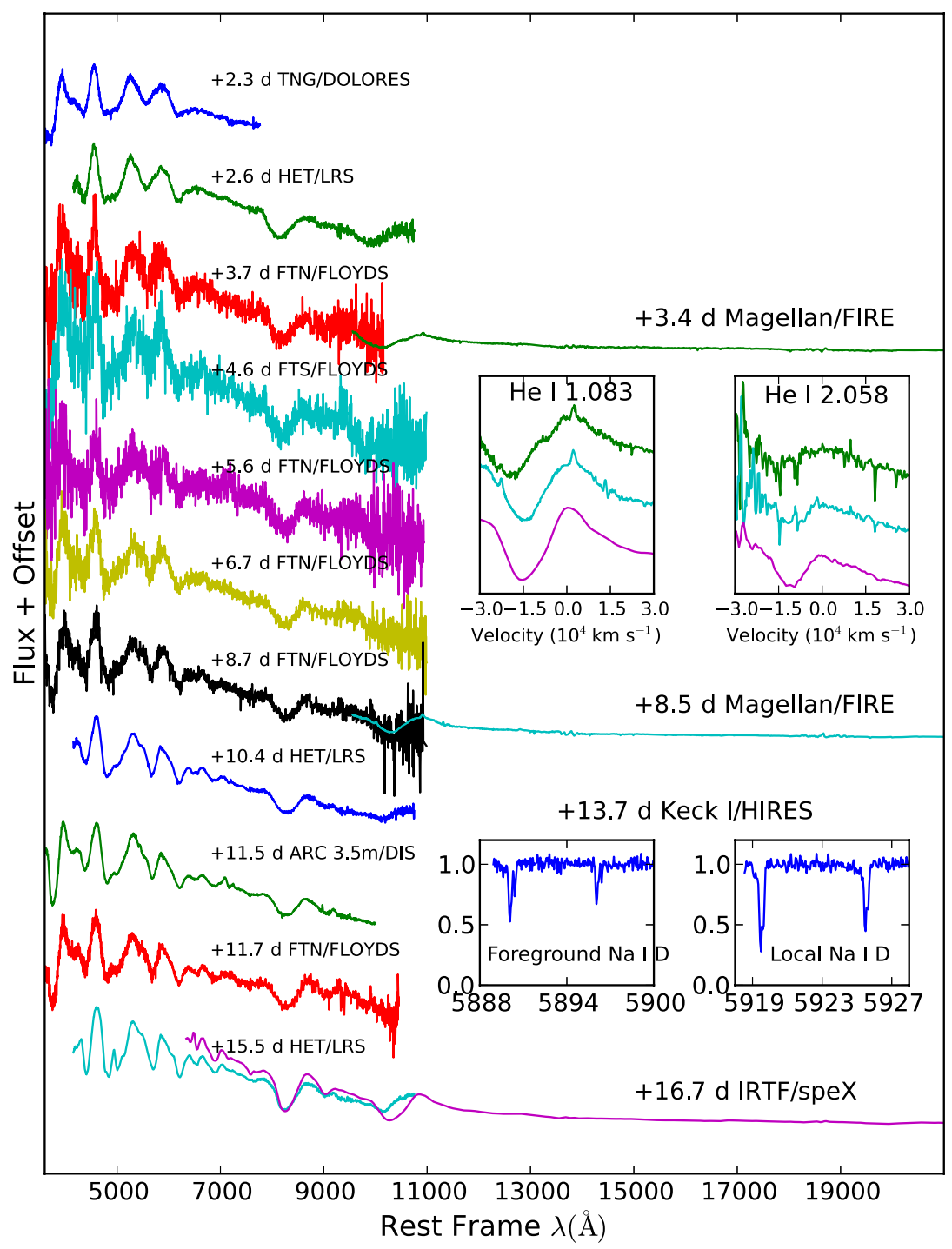

Figure 3. Spectral sequence of iPTF13bvn. Each spectrum is labeled with the observation date, the telescope, and the instrument. The upper two insets zoom-in to the infrared He I 10830 and He I 20581 lines. The lower two insets zoom-in to the Na I D doublet at high resolution.

(A color version of this figure is available in the online journal.)

Swift images, the host background is subtracted by using preexplosion reference images, while in the LCOGT images a loworder polynomial fit is used to remove the background. PSF photometry is then performed in all the images. Photometry in the $g^{\prime} r^{\prime} i^{\prime} z^{\prime}$ bands is calibrated to Sloan Digital Sky Survey stars and that in the $U B V R I$ bands is calibrated with Landolt standard stars. The multi-color light curve of iPTF13bvn is illustrated in Figure 2.

Low-resolution spectroscopic follow-up of iPTF13bvn was undertaken with the DOLORES low-resolution spectrograph on Telescopio Nazionale Galileo (TNG), the Marcario LowResolution Spectrograph (LRS; Hill et al. 1998) on the Hobby-Eberly Telescope (HET), the low-resolution, crossdispersed spectrograph FLOYDS (D. Sand et al., in preparation) on the robotic Faulkes Telescope (FT), the Dual Imaging Spectrograph (DIS) on the ARC $3.5 \mathrm{~m}$ telescope, the FoldedPort InfraRed Echellete (FIRE; Simcoe et al. 2013) on the $6.5 \mathrm{~m}$ Magellan telescope, and SpeX (Rayner et al. 2003) on the NASA Infrared Telescope Facility (IRTF). We also obtained a highresolution spectrum with High Resolution Echelle Spectrometer (HIRES; Vogt et al. 1994) on the $10 \mathrm{~m}$ Keck I telescope. The spectroscopic series of iPTF13bvn is displayed in Figure 3.
All photometry tables and spectroscopy data will be made public via WISeREP ${ }^{24}$ (Yaron \& Gal-Yam 2012).

\subsection{Analysis: Photometry}

In order to better constrain the explosion date, we fit a powerlaw model $f(t) \propto\left(t-t_{0}\right)^{\beta}$ to the P48 data of iPTF13bvn taken in the first five nights after explosion and constrain the parameters with the upper limits preceding the discovery. The best fit results in an explosion date $t_{0}=$ June 15.67 and a power-law index $\beta=1.01$ (Figure 2). The 95\% confidence levels for $t_{0}$ and $\beta$ are [Jun 15.50, Jun 15.76] and [0.923, 1.09], respectively. Note that the color evolution is minimal, suggesting that the $r$ band is a reasonable proxy for the bolometric light curve. iPTF13bvn peaked at $r=-16.6$ mag at about $t_{0}+18$ days.

A direct comparison of the iPTF13bvn $r$-band light curve with SN 1994I (Richmond et al. 1996), SN 2008D (Soderberg et al. 2008), PTF10vgv (Corsi et al. 2012), and PTF12gzk (Ben-Ami et al. 2012) is also shown in Figure 4. The Type Ic SN 1994I is much more luminous than $\mathrm{PTF} 13 \mathrm{bvn}$ while its rise rate is

\footnotetext{
24 http://www.weizmann.ac.il/astrophysics/wiserep/
} 


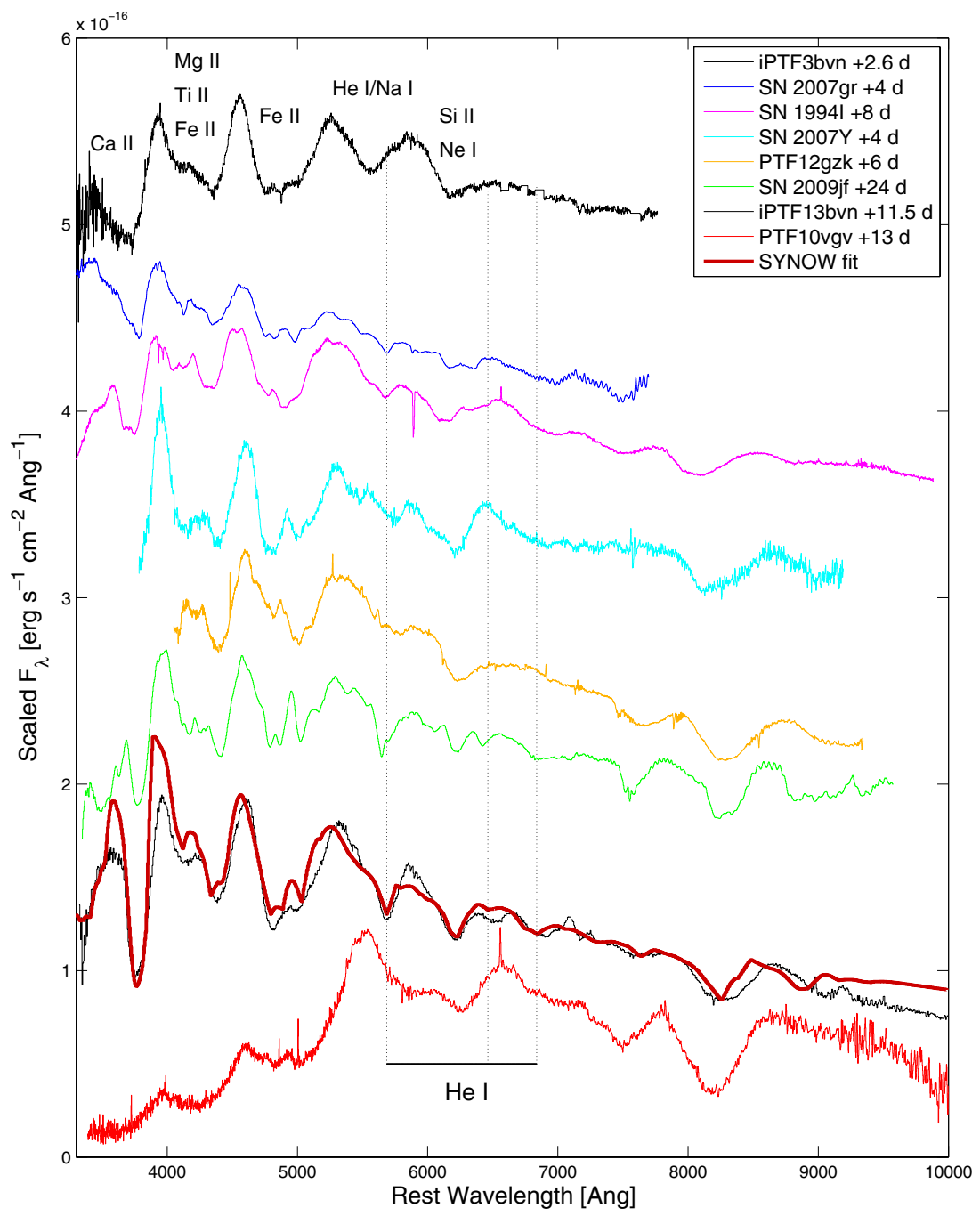

Figure 4. Spectra of iPTF13bvn (black) compared to other SNe Ib/c. All ages are reported in days since explosion. An early spectrum of iPTF13bvn shows strong similarity to early spectra of Type Ib/c SNe: SN 2007gr, Type Ic (Valenti et al. 2008); SN 1994I, Type Ic (Filippenko et al. 1995); SN 2007Y, Type Ib (Stritzinger et al. 2009). The early spectrum of the energetic Type Ic PTF12gzk (Ben-Ami et al. 2012) resembles that of iPTF13bvn, if a correction is made artificially for its remarkable blueshift. Later spectra of iPTF13bvn resemble the Type Ib SN 2009jf (Valenti et al. 2011). Despite the light curve similarity, the high velocity Type Ic PTF10vgv bears no spectral resemblance to iPTF13bvn.

(A color version of this figure is available in the online journal.)

less than that of iPTF13bvn. The Type Ib SN 2008D has a five day cooling phase after its shock breakout before it slowly rises again, while we do not detect any shock cooling signature from iPTF13bvn. The energetic Type Ic supernova PTF12gzk rises much faster than iPTF13bvn. Surprisingly, the closest match to the rise rate of iPTF13bvn through eight days is the high velocity Type Ic PTF10vgv (albeit about 2 mag fainter).

Following Piro \& Nakar (2013), we derive a constraint on the progenitor radius based on our detection limits on shock cooling. The inferred radius is sensitive to the "mean" velocity of the ejecta $v=\sqrt{2 E / M_{\mathrm{ej}}}$ where $E$ is the explosion energy and $M_{\mathrm{ej}}$ is the mass of the ejecta. Based on the measured photospheric velocities and line velocities (Section 4.3), we conservatively adopt the lowest velocity of $8000 \mathrm{~km} \mathrm{~s}^{-1}$. If the velocity was higher, the radius of the progenitor would be constrained to be even smaller (Piro \& Nakar 2013). The model also depends weakly on $E$ and thus we assume $E=10^{51} \mathrm{erg}$. In the right bottom panel of Figure 2, the predicted $r$-band light curves (gray lines) of shock cooling with various explosion times are also plotted. We find: if the explosion had happened earlier than $t_{0}$, the progenitor radius would have to be unreasonably small $\left(\approx 0.03 R_{\odot}\right)$ because the luminosity of the shock breakout is tightly constrained by non-detection on June 14. If the explosion happened at $t_{0}$, the progenitor radius $<1.5 R_{\odot}$. If the explosion happened at $t_{0}+0.5$ day, the progenitor radius $<5 R_{\odot}$. We conclude that the progenitor radius was no larger than a few solar radii, suggesting a stripped core such as WR stars.

\subsection{Analysis: Spectroscopy}

The strongest features in the early spectra are the $\mathrm{Ca}$ II $\mathrm{H}+\mathrm{K}$ and Ca II near-IR triplet absorption. The blends of Fe II lines at $\approx 5000 \AA$ and Fe II, Mg II and Ti II at $\approx 4400 \AA$ are also prominent. The local minimum at about $5500 \AA$ may be either $\mathrm{He}$ I or Na I and that at about $6200 \AA$ may be interpreted as either $\mathrm{Si}$ II or Ne I. At +2.6 days after explosion, a SYNOW fit gives a photospheric velocity of $10,000 \mathrm{~km} \mathrm{~s}^{-1}$ and a Ca II line velocity of $14,000 \mathrm{~km} \mathrm{~s}^{-1}$. By +11.5 days, these velocities evolve to 8000 and $10,000 \mathrm{~km} \mathrm{~s}^{-1}$ respectively.

Helium lines are not expected to be prominent in early spectra of Type Ib supernovae (Hachinger et al. 2012). We begin to 


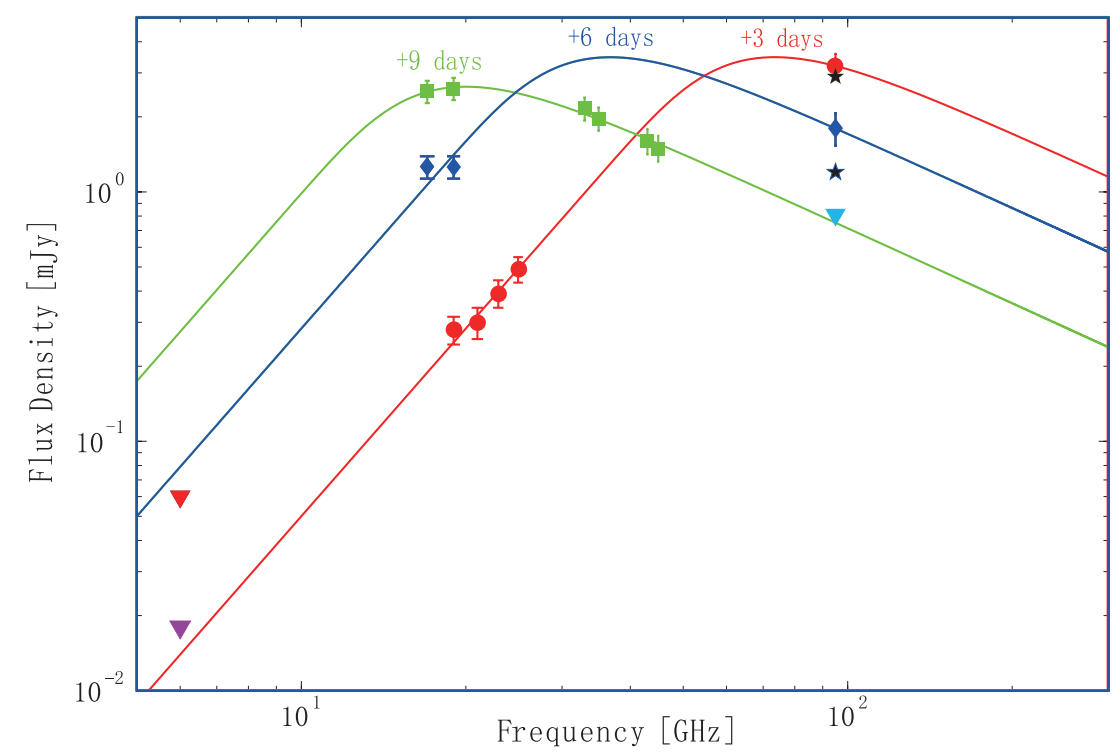

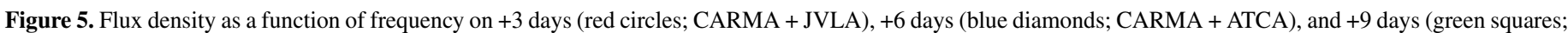

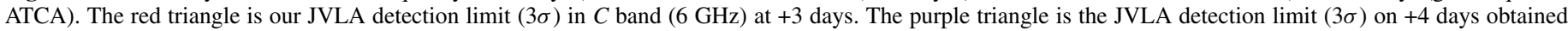

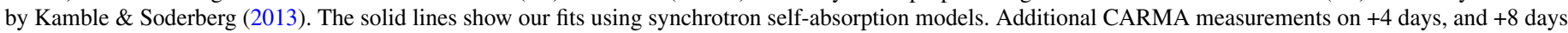
(black stars) and an upper limit on +10 days (cyan triangle) are shown.

(A color version of this figure is available in the online journal.)

see weak wiggles at the location of He I $(5876 \AA$, $6678 \AA$ and $7065 \AA$ ) in optical spectra after +10 days. However, SYNOW cannot fit a single expansion velocity to all the three lines simultaneously. In the IR spectra, we see a prominent $10200 \AA$ absorption feature, which may be explained by He I 10830 line blended with metal lines. The $20581 \AA$ doublet is often used to confirm the presence of He (Taubenberger et al. 2006; Marion et al. 2013). By +16.7 days, we unambiguously detect this feature (see the inset of Figure 3). Therefore, we classify iPTF13bvn as a Type Ib supernova (Milisavljevic et al. 2013; Kasliwal et al. 2013).

\section{RADIO FOLLOW-UP}

Promptly after discovery, we initiated radio follow-up observations at the Combined Array for Research in Millimeter-wave Astronomy (CARMA) ${ }^{25}$ and requested Director's Discretionary Time at the Jansky Very Large Array (JVLA). ${ }^{26}$ Our JVLA and CARMA data showed that the radio counterpart had a synchrotron self-absorption (SSA) spectrum. On being denied our request for additional JVLA observations, our colleagues were able to initiate Australia Telescope Compact Array (ATCA) ${ }^{27}$ observations. The JVLA data were reduced using AIPS with J1505+0326 as a phase calibrator and 3C286 as a flux calibrator. The CARMA data were reduced using MIRIAD (Sault et al. 1995) with J1549+026 as a phase calibrator and MWC349 as a flux calibrator. The ATCA data were reduced using MIRIAD with PKS B1921-293 as a bandpass calibrator, PKS B1508-055 as a phase calibrator, and PKS B1934-638 as a flux calibrator. All the radio data is presented in Figure 5.

We fit the multi-frequency multi-epoch data to the SSA model following Chevalier (1998). We assume that the electron energy distribution can be described by a power law $N(E) \propto E^{-3}$. The fitted models are also presented in Figure 5.

\footnotetext{
25 CARMA program c1127, PI: Horesh.

26 JVLA program 13A-531, PI: Horesh.

27 ATCA program C2707, PI: Hancock.
}

We note that the SSA model alone is consistent with all our data including the null detection in $C$ band on June 18 . However, on June 19.1, Kamble \& Soderberg (2013) observed iPTF13bvn with the JVLA in $C$ band and announced a null detection with an rms of $6 \mu \mathrm{Jy}$. Based on our SSA model, we estimate that Kamble \& Soderberg should have detected iPTF13bvn at a level of $\sim 5 \sigma$. Their null detection therefore may require additional free-free absorption at low frequencies. Based on inverse Compton scattering, we estimate the X-ray emission to be $10^{-15} \mathrm{erg} \mathrm{cm}^{-2} \mathrm{~s}^{-1}$. This is consistent with our Swift X-ray telescope (XRT) upper limit of $1.0 \times 10^{-13} \mathrm{erg} \mathrm{cm}^{-2} \mathrm{~s}^{-1}$ on June 17 (assuming a power-law spectrum with a photon index of 2.0). We note that Kong et al. (2013) reported an X-ray detection by co-adding the XRT data from June 17 to July 13. We reprocessed the XRT data from the first 10 days after the supernova explosion and resulted in a $3 \sigma$ upper limit of $4 \times 10^{-14} \mathrm{erg} \mathrm{cm}^{-2} \mathrm{~s}^{-1}$. This is also consistent with our expectation from inverse Compton scattering.

We find that the radio emission peaks at $\approx 73.5,37,20 \mathrm{GHz}$ with fluxes of $\approx 3.5,3.5,2.7 \mathrm{mJy}$ on June 18,21 and 24 , respectively. Following Chevalier (1998), we derive a shock wave radius $R_{s} \approx 0.7,1.4,2.3 \times 10^{15} \mathrm{~cm}$ and a magnetic field strength $B \approx 8.2,4.1,2.3 \mathrm{G}$. Using the explosion date determined in the optical data, we find a shock wave velocity of $2.7 \times 10^{4} \mathrm{~km} \mathrm{~s}^{-1}$, which is typical for $\mathrm{SNe} \mathrm{Ib/c} \mathrm{(Chevalier} \mathrm{\&}$ Fransson 2006).

We derive a mass-loading parameter $A \approx 1.3 \times 10^{12} \mathrm{~g} \mathrm{~cm}^{-1}$ (see Horesh et al. 2012 for equations). Assuming a typical wind velocity of $v_{w} \approx 1000 \mathrm{~km} \mathrm{~s}^{-1}$ from a WR star (Cappa et al. 2004), the progenitor mass-loss rate $\dot{M}=4 \pi A v_{w} \approx$ $3 \times 10^{-5} M_{\odot} \mathrm{yr}^{-1}$. This is consistent with the WR mass-loss rate in Cappa et al. (2004).

\section{CONCLUSION}

iPTF13bvn is a Type Ib supernova that exploded on June 15.67 and rose to maximum luminosity of $M_{R}=-16.6$ in about 18 days. The luminosity of the supernova in the first 
10 days is approximately proportional to $t^{-1}$. We identify a single progenitor candidate within a $2 \sigma$ error radius of $8.7 \mathrm{pc}$ in pre-explosion $H S T$ imaging. The candidate has an $M_{B}$ luminosity of $-5.52 \pm 0.39 \mathrm{mag}$ and a $B-I$ color of $0.25 \pm 0.25 \mathrm{mag}$. Future HST imaging, after the supernova fades away, will determine whether this is a single star, a binary, or a star cluster. The non-detection of shock cooling in our light curve constrains the progenitor radius to smaller than a few solar radii. Our radio detections suggest a shock wave with velocity of $2.7 \times 10^{4} \mathrm{~km} \mathrm{~s}^{-1}$ and a progenitor mass-loss rate of $3 \times 10^{-5} M_{\odot} \mathrm{yr}^{-1}$.

We conclude that the pre-explosion photometry of the detected candidate, the radius constraint based on absence of shock cooling, and the mass-loss rate derived from radio are all consistent with a WR progenitor.

We thank A. L. Piro for valuable discussions. We thank the following people for co-operating with our target of opportunity or queue observations: M. Roth (Magellan), A. Hartuynan (TNG), J. Johnson (Keck), and J. Caldwell (HET). We thank A. Howard and $\mathrm{H}$. Isaacson for HIRES data reduction. We thank R. Campbell, Hien Tran, and S. Tendulkar for helping with OSIRIS LGS-AO observation and data reduction. We thank P. Vreeswijk for assisting with HST image registration. We thank J. Vinko, R. Foley, B. Kirshner, D. Perley, A. Corsi, and K. Mooley as proposal co-Is. We thank J. Swift, B. Montet, M. Bryan, R. Jensen-Clem, D. Polishook, and S. Tinyanont for assisting with observations.

M.M.K. acknowledges generous support from the Hubble Fellowship and Carnegie-Princeton Fellowship. J.M.S. is supported by an NSF Astronomy and Astrophysics Postdoctoral Fellowship under award AST-1302771. N.D. acknowledges the Hubble Fellowship. Research by A.G.Y. and his group was supported by grants from the ISF, BSF, GIF, Minerva, the EU/FP7 via an ERC grant and the Kimmel award. The research of J.C.W. is supported by NSF Grant AST 11-09801.

The National Energy Research Scientific Computing Center, supported by the Office of Science of the U.S. Department of Energy, provided staff, computational resources, and data storage for this project. The Australia Telescope is funded by the Commonwealth of Australia for operation as a National Facility managed by CSIRO. This research has been supported by the Australian Research Council through Super Science Fellowship grant FS100100033. The Centre for All-sky Astrophysics is an Australian Research Council Centre of Excellence, funded by grant CE110001020. The National Radio Astronomy Observatory is a facility of the National Science Foundation operated under cooperative agreement by Associated Universities, Inc. Ongoing CARMA development and operations are supported by the National Science Foundation under a cooperative agreement, and by the CARMA partner universities.

\section{REFERENCES}

Arcavi, I., Cenko, S. B., Gal-Yam, A., \& Ofek, E. 2013a, ATel, 5146, 1 Arcavi, I., Ofek, E., Cao, Y., et al. 2013b, ATel, 5152, 1

Ben-Ami, S., Gal-Yam, A., Filippenko, A. V., et al. 2012, ApJL, 760, L33

Bersten, M. C., Benvenuto, O. G., Nomoto, K., et al. 2012, ApJ, 757, 31

Brown, T. M., Baliber, N., Bianco, F., et al. 2013, PASP, in press (arXiv:1305.2437)

Cao, Y., Gorbikov, E., Arcavi, I., et al. 2013, ATel, 5137, 1

Cappa, C., Goss, W. M., \& van der Hucht, K. A. 2004, AJ, 127, 2885

Cenko, S. B., Fox, D. B., Moon, D.-S., et al. 2006, PASP, 118, 1396

Chevalier, R. A. 1998, ApJ, 499, 810

Chevalier, R. A., \& Fransson, C. 2006, ApJ, 651, 381

Corsi, A., Ofek, E. O., Gal-Yam, A., et al. 2012, ApJL, 747, L5

Crockett, R. M., Smartt, S. J., Eldridge, J. J., et al. 2007, MNRAS, 381, 835

Dessart, L., Hillier, D. J., Li, C., \& Woosley, S. 2012, MNRAS, 424, 2139

Dolphin, A. E. 2000, PASP, 112, 1383

Eldridge, J. J., Fraser, M., Smartt, S. J., Maund, J. R., \& Crockett, R. M. 2013, arXiv: 1301.1975

Filippenko, A. V. 1997, ARA\&A, 35, 309

Filippenko, A. V., Barth, A. J., Matheson, T., et al. 1995, ApJL, 450, L11

Hachinger, S., Mazzali, P. A., Taubenberger, S., et al. 2012, MNRAS, 422, 70

Hill, G. J., Nicklas, H. E., MacQueen, P. J., et al. 1998, Proc. SPIE, 3355, 375

Horesh, A., Kulkarni, S. R., Fox, D. B., et al. 2012, ApJ, 746, 21

Kamble, A., \& Soderberg, A. 2013, ATel, 5154, 1

Kasen, D. 2010, ApJ, 708, 1025

Kasliwal, M. M., Degenaar, N., \& Polishook, D. 2013, ATel, 5151, 1

Kong, A. K. H., Li, K. L., \& Ofek, E. 2013, ATel, 5210, 1

Larkin, J., Barczys, M., Krabbe, A., et al. 2006, Proc. SPIE, 6269, 62691A

Law, N. M., Kulkarni, S. R., Dekany, R. G., et al. 2009, PASP, 121, 1395

Li, W., Chornock, R., Leaman, J., et al. 2011, MNRAS, 412, 1473

Marion, G. H., Vinko, J., Kirshner, R. P., et al. 2013, arXiv:1303.5482

Milisavljevic, D., Fesen, R., Pickering, T., et al. 2013, ATel, 5142, 1

Nakar, E., \& Sari, R. 2010, ApJ, 725, 904

Ofek, E. O., Laher, R., Law, N., et al. 2012, PASP, 124, 62

Piro, A. L., \& Nakar, E. 2013, ApJ, 769, 67

Poznanski, D., Prochaska, J. X., \& Bloom, J. S. 2012, MNRAS, 426, 1465

Rabinak, I., \& Waxman, E. 2011, ApJ, 728, 63

Rayner, J. T., Toomey, D. W., Onaka, P. M., et al. 2003, PASP, 115, 362

Richmond, M. W., van Dyk, S. D., Ho, W., et al. 1996, AJ, 111, 327

Sault, R. J., Teuben, P. J., \& Wright, M. C. H. 1995, in ASP Conf. Ser. 77, Astronomical Data Analysis Software and Systems IV, ed. R. A. Shaw, H. E. Payne, \& J. J. E. Hayes (San Francisco, CA: ASP), 433

Schlafly, E. F., \& Finkbeiner, D. P. 2011, ApJ, 737, 103

Simcoe, R. A., Burgasser, A. J., Schechter, P. L., et al. 2013, PASP, 125, 270

Soderberg, A. M., Berger, E., Page, K. L., et al. 2008, Natur, 453, 469

Stritzinger, M., Mazzali, P., Phillips, M. M., et al. 2009, ApJ, 696, 713

Taubenberger, S., Pastorello, A., Mazzali, P. A., et al. 2006, MNRAS, 371, 1459

Tully, R. B., Rizzi, L., Shaya, E. J., et al. 2009, AJ, 138, 323

Tuthill, P. G., Monnier, J. D., \& Danchi, W. C. 1999, Natur, 398, 487

Valenti, S., Elias-Rosa, N., Taubenberger, S., et al. 2008, ApJL, 673, L155

Valenti, S., Fraser, M., Benetti, S., et al. 2011, MNRAS, 416, 3138

Vogt, S. S., Allen, S. L., Bigelow, B. C., et al. 1994, Proc. SPIE, 2198, 362

Wizinowich, P. L., Chin, J., Johansson, E., et al. 2006, Proc. SPIE, 6272, 627209

Yaron, O., \& Gal-Yam, A. 2012, PASP, 124, 668

Yoon, S.-C., Gräfener, G., Vink, J. S., Kozyreva, A., \& Izzard, R. G. 2012, A\&A, 544, L11 